\title{
Comparison of the McGrath MAC EMS Videolaryngoscope with a Conventional Laryngoscope for Standard and Difficult Airway Intubation: A Randomized, Cross-over, Simulation Trial
}

\author{
(1) Semih Korkut ${ }^{1}$, (1) Lukasz Szarpak², (1) Togay Evrin ${ }^{3}$, (1) Jacek Smereka ${ }^{4}$, (1) Burak Katipoğlu³, (1) Damian Gorczyca ${ }^{2}$ \\ ${ }^{1}$ Clinic of Emergency Medicine, İstanbul Kartal Dr. Lütfi Kırdar Training and Research Hospital, İstanbul, Turkey \\ 2Medical Simulation Center, Lazarski University, Warsaw, Poland \\ ${ }^{3}$ Clinic of Emergency Medicine, Ufuk University Faculty of Medicine, Dr. Rıdvan Ege Training and Research Hospital, Ankara, Turkey \\ ${ }^{4}$ Department of Emergency Medical Service, Wroclaw Medical University, Wroclaw, Poland
}

\begin{abstract}
Aim: Tracheal intubation is challenging in emergency medicine, especially in pre-hospital settings, owing to problems connected with patients, intubator, and the environment. The study aimed to compare the effectiveness of endotracheal intubation performed by paramedics with the use of direct laryngoscopy and the McGrath MAC videolaryngoscope in standard and difficult airway settings.

Materials and Methods: The study was designed as a prospective, randomized, cross-over simulation study. It involved 52 paramedics. The participants performed endotracheal intubation in two scenarios: Normal airway and difficult airway, achieved by inflation of the simulator tongue to the level of difficulty determined by an independent instructor at grade 3 on the Cormack-Lehane scale. The primary endpoint was the success of endotracheal intubation; intubation time constituted the secondary endpoint.

Results: The median age of participants was 24 years [interquartile range (IQR): 23-25], and the median work experience was 0.5 years (IQR: $0-1)$. In normal airway (scenario A), the effectiveness of the first intubation attempt was $92.3 \%$ for MAC and $96.2 \%$ for McGrath ( $p=0.724$ ). The median intubation time for MAC was 19 (IQR: 14-21.5) seconds and was statistically significantly longer than 16 (12.5-20) seconds for McGrath ( $p=0.047$ ). The ease of intubation was 18 (IQR: 10-20) points for McGrath and 25 (IQR: 16-27) points for MAC ( $p=0.035)$. In difficult airway (scenario B), the efficacy of the first intubation attempt for MAC and McGrath equaled $40.4 \%$ and $82.7 \%$, respectively. The median intubation time for McGrath was 19 (IQR: 14-27.5) seconds and was significantly shorter than 25 (IQR: 24-39) seconds for MAC ( $p=0.007)$. The degree of glottis visualization was statistically significantly better in McGrath than in MAC $(p<0.001)$. The ease of intubation was 25 (IQR: 20-32) points for McGrath and 49 (IQR: 34-58) points for MAC ( $p<0.001)$. Overall, $96.2 \%$ of subjects intubating in difficult airway conditions chose the McGrath videolaryngoscope as their preferred intubation method as compared with MAC.

Conclusion: In the simulation study, endotracheal intubation in difficult airway conditions performed by paramedics using McGrath video laryngoscopy was associated with shorter procedure duration, better glottis visualization, and higher efficacy of the first intubation attempt. The study showed that paramedics were able to intubate using the McGrath videolaryngoscope with higher efficiency than in the case of a standard Macintosh laryngoscope after a short training.
\end{abstract}

Keywords: Airway management, McGrath MAC EMS, difficult airway, videolaryngoscopy, simulation

\section{Introduction}

Tracheal intubation is the gold standard for airway management. The advantages of endotracheal intubation include reliable ventilation, separation of the airway from the gastrointestinal tract, significant reduction of regurgitation risk, the possibility to suction the respiratory tract, and to ventilate in further patient care conditions, including intensive care units $(1,2)$. Endotracheal intubation can be performed with many techniques, but direct 
laryngoscopy is usually applied. This method, however, requires appropriate experience, and many intubation attempts to maintain an adequate level of experience and manual skills (3). Because of the risk of severe and potentially fatal complications associated with endotracheal intubation, it is necessary to consider the experience, necessity of endotracheal intubation, technical possibilities, and alternative methods. Considering the above problems, the quality of intubation, including the effectiveness of the first intubation attempt, the assessment of complication risk, and the total duration of intubation procedure have been studied for several years $(4,5)$.

Tracheal intubation is challenging in emergency medicine, especially in pre-hospital settings, owing to problems connected with the patient, intubator, and environment $(6,7)$. Performing endotracheal intubation in the case of sudden cardiac arrest in a patient with difficult airways by a relatively inexperienced professional in unfavorable environmental conditions is associated with a very high risk of esophageal intubation and other complications (8).

The development of videolaryngoscope marked significant progress in anesthesiology and emergency medicine $(9,10)$. The devices enable endotracheal intubation even when the entrance to the larynx cannot be directly visualized (11). Videolaryngoscope is used not only in elective cases in the operating theatre during difficult intubation but also in emergency medicine (12). Many surveys, including manikin studies and human studies $(13,14)$, indicate that the duration of endotracheal intubation is shorter compared with direct laryngoscopy, especially in difficult airways, as this is not always confirmed in the case of normal airways (14). Although in critical care settings, in patients undergoing endotracheal intubation, videolaryngoscopy improves glottic visualization, it does not appear to increase procedural success or decrease complications (15). Large clinical multicenter studies were carried out to compare videolaryngoscope and direct laryngoscopy (14).

One of the available videolaryngoscope on the market is the McGrath MAC EMS videolaryngoscope. It combines direct laryngoscopy and videolaryngoscopy in one device. Many intubators, especially emergency medical service providers, have greater experience with direct laryngoscopy. McGrath MAC EMS enables both direct visualization and videolaryngoscopy and can facilitate intubation.

The study aimed to compare the effectiveness of endotracheal intubation performed by paramedics with the use of direct laryngoscopy and the McGrath MAC videolaryngoscope in standard and difficult airway settings.

\section{Materials and Methods}

The study protocol was approved by the Institutional Review Board of the Polish Society of Disaster Medicine (number: 23.02.2019.IRB, on 12.02.2019). The study is a continuation of research on identifying an alternative intubation method to direct laryngoscopy (16-19). The trial was designed as a prospective, randomized, cross-over manikin study. Written informed consent was obtained from all 52 paramedics taking part in the study. All subjects had $<1$ year of experience and had performed $<10$ endotracheal intubations in real patients. None of them had previously had any experience with the use of videolaryngoscopy.

\section{Study Design}

Before the study, all participants attended an airway management workshop, which included endotracheal intubation with the use of direct laryngoscopy and videolaryngoscopy. Two types of laryngoscopes were used in the study:

1. Standard laryngoscope with Macintosh blade size 3 (gold standard; Heine Optotechnik, Herrsching, Germany);

2. McGrath MAC EMS videolaryngoscope (Aircraft Medical Limited, Edinburgh, Scotland; Figure 1).

After an instruction showing the correct intubation technique, the subjects took part in 30 minute practical workshops with the use of the studied devices. The training took place in normal airway conditions; an airway manikin Ambu Airway Man (Ambu, Copenhagen, Denmark) was involved.

During the target study, an advanced adult SimMan 3G simulator (Laerdal, Stavanger, Norway) was applied to simulate a patient requiring endotracheal intubation. The simulator was placed on the floor in a room with daylight. The study participants performed endotracheal intubation in 2 scenarios:

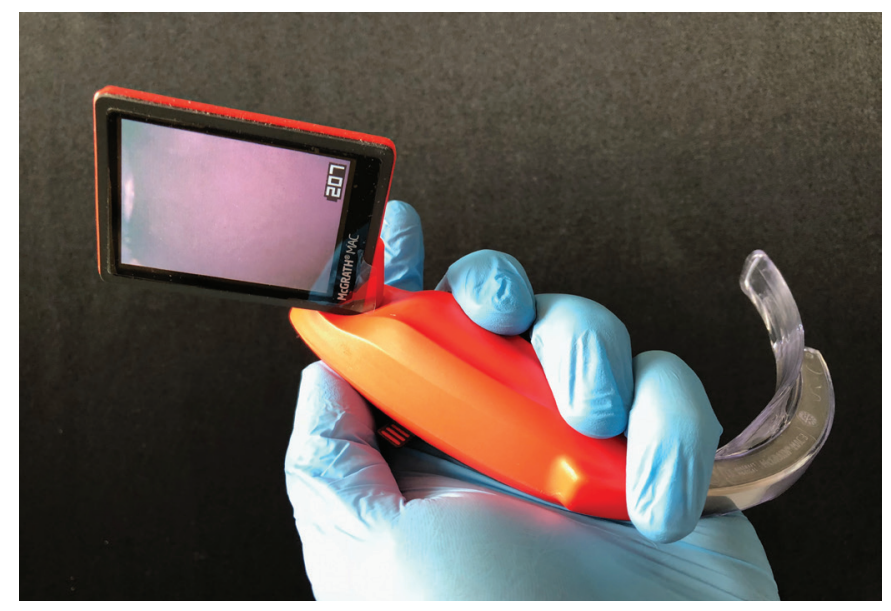

Figure 1. MCGrath MAC EMS videolaryngoscope 


\section{Scenario A: Normal airway.}

2. Scenario B: Difficult airway. Difficult airways were achieved by inflation of the simulator tongue to the level of difficulty determined by an independent instructor at grade 3 on the Cormack-Lehane scale (20).

A standard intubation stylet was used for all intubations. The participants had a maximum of 3 endotracheal intubation attempts. The order of both subjects and research methods were random. For this purpose, Research Randomizer (randomizer. org) was used to divide the participants into 4 groups: the first one performed endotracheal intubation by using MAC during scenario $\mathrm{A}$, the second one by using MAC during scenario $B$, the third one by using McGrath in scenario A, and the fourth one by using McGrath during scenario B. After the intubation attempts with a given method, the participants had a 10-minute break and then intubated with a different technique. The detailed randomization procedure is presented in Figure 2.

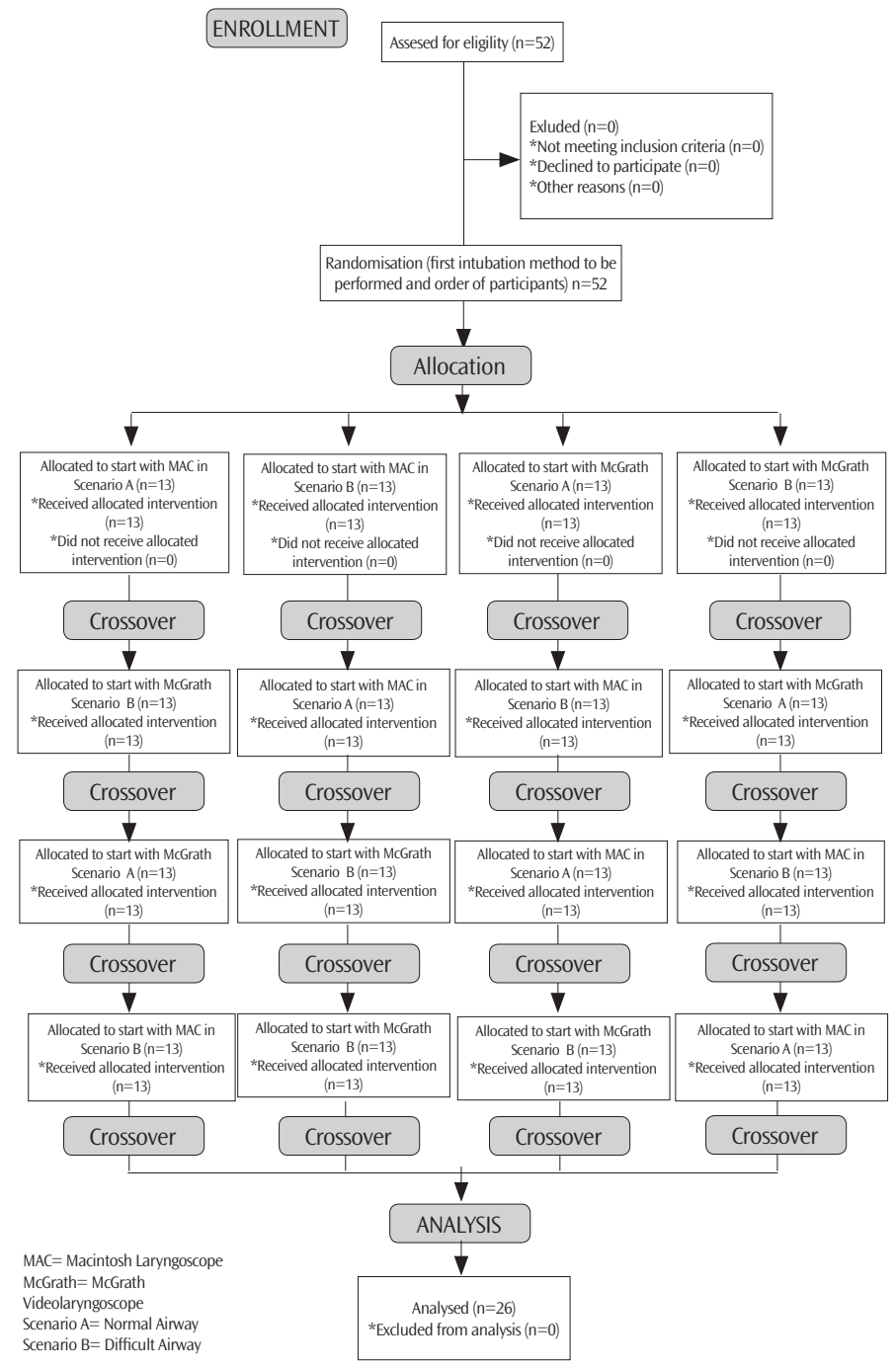

Figure 2. Randomization flow chart

\section{Measurements}

The primary end point was the success of endotracheal intubation, confirmed by a researcher by the ability to ventilate the manikin lungs with a self-inflating bag connected to the endotracheal tube. The following criteria were defined for a failed intubation: more than three unsuccessful intubation attempts, intubation procedure exceeding 120 seconds, unrecognized esophageal intubation.

Intubation time, the secondary endpoint, was defined as the time from the first insertion of the blade between the teeth until the first successful ventilation of the lungs recorded with a stopwatch of a mobile phone. Vocal cord visualization was assessed by Cormack-Lehane grade (20). The paramedics' subjective opinion on the ease of use of each device was measured with a visual analog scale ranging from 1 (extremely easy) to 100 (extremely difficult). Finally, the participants were asked to indicate the device that they would prefer in a real-life emergency intubation setting.

\section{Statistical Analysis}

All analyses were performed with the STATISTICA 13.3EN statistical package (Tibco, Tulsa, OK, USA). We determined the sample size based on a previous study calculation (21). In the context of using a paired 2-sided t-test, accepting an $\alpha$ risk of $p<0.05$, powered to $80 \%, 46$ participants were required.

Data are presented as number (percentage) or median [interquartile range (IQR)]. Non-parametric tests were used for the data that did not have a normal distribution. The Wilcoxon test for paired observations was applied to compare the different times and to determine the statistical difference for each group. McNemar test served to evaluate the differences in intubation success rates and Cormack-Lehane grade. The ease-of-use scale, as well as the preferred airway device, were assessed with the Stuart-Maxwell test. All tests were 2-sided, and the value of $\mathrm{p}<0.05$ was considered statistically significant.

\section{Results}

The study involved 52 paramedics with a median age of 24 years (IQR: 23-25) and a median work experience of 0.5 years (IQR: 0-1). None of the subjects had experience with videolaryngoscopy; however, they all had clinical experience with direct laryngoscopy.

\section{Normal Airway (Scenario A)}

The effectiveness of the first intubation attempt was $92.3 \%$ for MAC and $96.2 \%$ for McGrath $(p=0.724)$. The median intubation time for MAC was 19 (IQR: 14-21.5) seconds and was statistically significantly longer than 16 (12.5-20) seconds for McGrath $(p=0.047$; Figure 3). The analysis of the study material did not 
reveal statistically significant differences in the degree of glottis visualization expressed on the Cormack-Lehane scale (Table 1). The ease of intubation was 18 (IQR: 10-20) points for McGrath and 25 (IQR: 16-27) points for MAC ( $p=0.035$; Figure 4). Overall, 31 subjects, which accounted for $59.6 \%$ of the study group, indicated McGrath as their preferred method of laryngoscopy.

\section{Difficult Airway (Scenario B)}

A detailed summary of scenario B results is presented in Table 2. The efficacy of the first intubation attempt for MAC and McGrath equaled $40.4 \%$ and $82.7 \%$, respectively. The median intubation time for McGrath was 19 (IQR: 14-27.5) seconds and was significantly shorter than 25 (IQR: 24-39) seconds for MAC $(p=0.007$; Figure 3$)$. The degree of glottis visualization was statistically significantly better in McGrath than in MAC $(p<0.001)$. The ease of intubation was 25 (IQR: 20-32) points for McGrath, and 49 (IQR: 34-58) points for MAC ( $<<0.001$; Figure 4). Overall, 50 (96.2\%) subjects intubating in difficult airway conditions chose the McGrath videolaryngoscope as their preferred intubation method as compared with MAC.

\section{Discussion}

The simulation study showed statistically significant differences in the duration of the intubation procedure and the efficacy of the first intubation attempt when using a standard Macintosh blade laryngoscope and the McGrath MAC EMS videolaryngoscope.

Endotracheal intubation in pre-hospital settings can be challenging even for experienced intubators $(22,23)$. It should be remembered that each intubation performed within an emergency medical service is emergency intubation (24). The lack of information on patient status and medical history, inability to conduct an anesthesia consultation in many cases, as well as the lack of knowledge of the recent meal, make each patient in the pre-hospital conditions and the emergency department considered as a patient with difficult airways (25).

Table 1. Normal airway (scenario A)

\begin{tabular}{|c|c|c|c|}
\hline Parameter & Direct laryngoscopy (MAC) & Videolaryngoscopy (McGrath) & p \\
\hline Overall success rate, $\mathrm{n}(\%)$ & $52(100 \%)$ & $52(100 \%)$ & NS \\
\hline \multicolumn{4}{|l|}{ Number of intubation attempts, $\mathbf{n}(\%)$} \\
\hline 1 & $48(92.3 \%)$ & $50(96.2 \%)$ & \multirow{3}{*}{ NS } \\
\hline 2 & $4(7.7 \%)$ & $2(3.8 \%)$ & \\
\hline 3 & - & - & \\
\hline The median time to intubation, $\mathrm{S}(\mathrm{IQR})$ & $19(14-21.5)$ & $16(12.5-20)$ & 0.047 \\
\hline \multicolumn{4}{|l|}{ Cormack-Lehane grade, $\mathrm{n}(\%)$} \\
\hline 1 & $46(88.5 \%)$ & $52(100 \%)$ & \multirow{4}{*}{ NS } \\
\hline 2 & $6(11.5 \%)$ & - & \\
\hline 3 & - & - & \\
\hline 4 & - & - & \\
\hline Ease of intubation, points on a 1-100 scale (IQR) & $25(16-27)$ & $18(10-20)$ & 0.035 \\
\hline Preferred airway device, n/52 overall & $21 / 52$ & $31 / 52$ & 0.011 \\
\hline
\end{tabular}

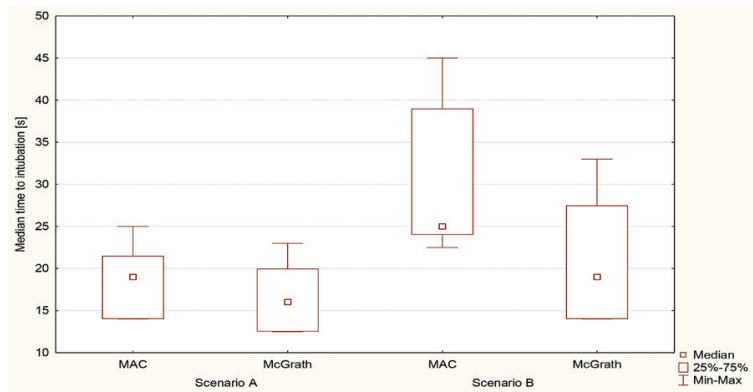

Figure 3. Median intubation time

Min: Minimum, Max: Maximum, MAC: Macintosh laryngoscope

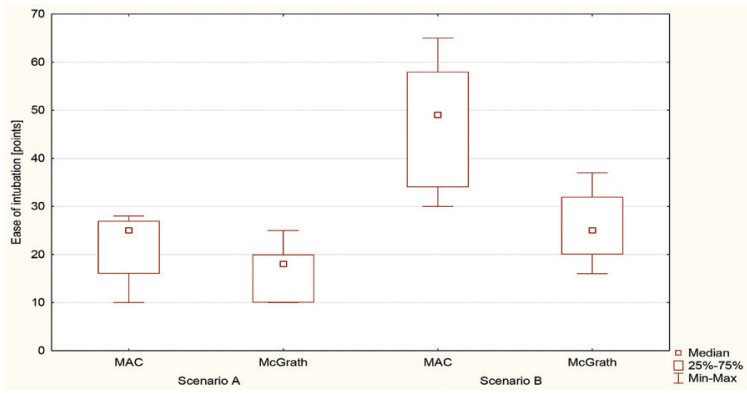

Figure 4. Ease of intubation

Min: Minimum, Max: Maximum, MAC: Macintosh laryngoscope 
Table 2. Difficult airway (scenario B)

\begin{tabular}{|c|c|c|c|}
\hline Parameter & Direct laryngoscopy (MAC) & Videolaryngoscopy (McGrath) & $\mathbf{p}$ \\
\hline Overall success rate, $\mathrm{n}(\%)$ & $52(100 \%)$ & $52(100 \%)$ & NS \\
\hline \multicolumn{4}{|l|}{ Number of intubation attempts, $\mathbf{n}(\%)$} \\
\hline 1 & $21(40.4 \%)$ & $43(82.7 \%)$ & \multirow{3}{*}{$<0.001$} \\
\hline 2 & $31(59.6 \%)$ & $9(17.3 \%)$ & \\
\hline 3 & - & - & \\
\hline The median time to intubation, $\mathrm{S}(\mathrm{IQR})$ & $25(24-39)$ & $19(14-27.5)$ & 0.007 \\
\hline \multicolumn{4}{|l|}{ Cormack-Lehane grade, $\mathrm{n}(\%)$} \\
\hline 1 & - & $50(96.2 \%)$ & \multirow{4}{*}{$<0.001$} \\
\hline 2 & $2(3.8 \%)$ & $2(3.8 \%)$ & \\
\hline 3 & $48(92.3 \%)$ & - & \\
\hline 4 & $2(3.8 \%)$ & - & \\
\hline Ease of intubation, points on a 1-100 scale (IQR) & $49(34-58)$ & $25(20-32)$ & $<0.001$ \\
\hline Preferred airway device, $\mathrm{n} / 52$ overall & $2 / 52$ & $50 / 52$ & $<0.001$ \\
\hline
\end{tabular}

In our study, the efficacy of the first intubation attempt and the total efficacy of intubation under normal airway were comparable for direct laryngoscopy and McGrath videolaryngoscopy. Additionally, in normal airway conditions, the glottis visualization was slightly better with McGrath than with Macintosh. Liu et al. (26) also indicate that the use of the McGrath laryngoscope by inexperienced anesthesiologists may improve the visibility of the glottis and the ease of the procedure and reduce the number of complications compared with direct laryngoscopy. Walker et al. (27) found no advantages of using the McGrath laryngoscope for uncomplicated tracheal intubation, but it is worth mentioning that anesthesiologists in their study performed intubation. On the other hand, studies conducted by Szarpak et al. (21) under normal airway conditions showed that the overall effectiveness of the UEScope and Macintosh laryngoscope was comparable, but in the case of the videolaryngoscope, paramedics were less likely to exert pressure on the teeth. In a prospective cohort study conducted at a simulation center of a university-based, tertiary care hospital, Kaki et al. (28) indicated that videolaryngoscope was better than the regular Macintosh when used by novice medical students for oral and nasal intubation in a manikin. Similar conclusions were expressed by other researchers (29).

Difficult airways can result from a variety of causes, including tongue edema, cervical spine immobilization, or difficult access to the patient. In all these situations, it is expected that the effectiveness of the first intubation attempt will be reduced, and the duration of the procedure will be prolonged with a MAC $(12,30,31)$. In the study, both the efficacy of the first intubation attempt and the duration of the procedure were significantly better with a videolaryngoscope than with a standard Macintosh blade laryngoscope.
In a study by Owada et al. (32) in which 20 anesthesiologists performed intubation in difficult airway conditions of a pediatric patient showed that the subjects were able to perform intubations using an Airtraq videolaryngoscope with a higher success rate, better visibility, and less dental trauma. In turn, Suzuki et al. (12), in their retrospective cohort study, indicated that the Pentax and McGrath laryngoscopes allowed for significantly higher emergency endotracheal intubation first-pass success rates compared with the Macintosh laryngoscope, especially among non-expert operators. Yoo et al. (33) revealed an advantage of McGrath and Macintosh laryngoscopes for double-lumen endobronchial tube intubation in patients with manual in-line stabilization.

Another significant result of the research is the fact that paramedics were able to intubate using a videolaryngoscope after short training effectively. In the case of direct laryngoscopy, the learning curve, as shown by numerous studies, illustrates 4375 attempts required for achieving successful intubation (34-36). The present study indicates an acceleration of the learning curve with videolaryngoscopy, and this is also confirmed in studies by Baciarello et al. (37), Aghamohammadi et al. (38), and other authors $(13,39,40)$.

Among the limitations of the conducted study, one can mention, among others, the fact that it was carried out in the conditions of medical simulation and not real rescue operations; this was, however, a deliberate action because medical simulation allows standardizing the performed procedures $(41,42)$ fully. Another reason for choosing medical simulation was that only this method allows conducting randomized cross-over studies without a potential detriment to the health and life of the patient 
(43). A further limitation is that only paramedics were involved in the study. Nevertheless, it is paramedics acting in emergency medical service settings who may meet the need for endotracheal intubation relatively often. Therefore, the evaluation of their skills, as well as the search for methods of intubation alternative to direct laryngoscopy, pose a challenge to researchers. The study also has its strong points. Among them are its randomized, crossover character and the use of two methods of laryngoscopy.

\section{Conclusion}

In the simulation study, endotracheal intubation in difficult airway conditions performed by paramedics using McGrath video laryngoscopy was associated with shorter procedure duration, better glottis visualization, and higher efficacy of the first intubation attempt. The study showed that after a short training, paramedics were able to intubate using the McGrath videolaryngoscope with higher efficiency than in the case of a standard Macintosh laryngoscope.

\section{Ethics}

Ethics Committee Approval: The study protocol was approved by the Institutional Review Board of the Polish Society of Disaster Medicine (approval no. 23.02.2019.IRB, 12.02.2019). Informed Consent: Written informed consent was obtained from all 52 paramedics taking part in the study.

Peer-review: Externally and internally peer-reviewed.

\section{Authorship Contributions}

Surgical and Medical Practices: L.S., J.S., D.G., Concept: L.S., T.E., Design: L.S., T.E., Data Collection or Processing: L.S., J.S., D.G., Analysis or Interpretation: L.S., Literature Search: S.K., J.S., B.K., D.G., Writing: S.K., L.S., T.E., J.S., B.K., D.G.

Conflict of Interest: No conflict of interest was declared by the authors.

Financial Disclosure: The authors declared that this study received no financial support.

\section{References}

1. Artime CA, Hagberg CA. Is there a gold standard for management of the difficult airway? Anesthesiol Clin. 2015;33:233-40.

2. Madziala A, Evrin T, Wieczorek W, Kaminska H, Nadolny K, Ladny JR, et al. Can the face-to-face intubation technique be used during cardiopulmonary resuscitation? A prospective, randomized, crossover manikin trial. Disaster Emerg Med J. 2017;2:145-9.

3. Markic S. Endotracheal tube ETView as a tool for airway management. Disaster Emerg Med J. 2018;3:152-3.

4. Hypes C, Sakles J, Joshi R, Greenberg J, Natt B, Malo J, et al. Failure to achieve first attempt success at intubation using video laryngoscopy is associated with increased complications. Intern Emerg Med. 2017;12:1235-43.
5. Klosiewicz T, Sip M, Zalewski R, Zielinski M. Alternative of endotracheal intubation for paramedics to provide direct laryngoscopy. The randomized manikin trial. Disaster Emerg Med J. 2017;2:142-4.

6. Sakles JC, Chiu S, Mosier J, Walker C, Stolz U. The importance of first pass success when performing orotracheal intubation in the emergency department. Acad Emerg Med. 2013;20:71-8.

7. Gawel W, Kaminska H, Wieczorek W. UEScope as a method of endotracheal intubation of trauma patient. Disaster Emerg Med J. 2017;2:175-6.

8. Sakles JC, Javedani PP, Chase E, Garst-Orozco J, Guillen-Rodriguez JM, Stolz $U$. The use of a video laryngoscope by emergency medicine residents is associated with a reduction in esophageal intubations in the emergency department. Acad Emerg Med. 2015;22:700-7.

9. Ladny JR, Smereka J, Szarpak L. Comparison of the Trachway video intubating stylet and Macintosh laryngoscope for endotracheal intubation. Preliminary data. Am J Emerg Med. 2017;35:574-5.

10. Szarpak L. Laryngoscopes for difficult airway scenarios: a comparison of the available devices. Expert Rev Med Devices. 2018;15:631-43.

11. Monette DL, Brown CA, Benoit JL, McMullan JT, Carleton SC, Steuerwald MT, et al. The impact of video laryngoscopy on the clinical learning environment of emergency medicine residents: a report of 14,313 intubations. AEM Educ Train. 2019;3:156-62

12. Suzuki K, Kusunoki S, Tanigawa K, Shime N. Comparison of three video laryngoscopes and direct laryngoscopy for emergency endotracheal intubation: a retrospective cohort study. BMJ Open. 2019;9:e024927.

13. Ladny JR, Sierzantowicz R, Kedziora J, Szarpak L. Comparison of direct and optical laryngoscopy during simulated cardiopulmonary resuscitation. Am J Emerg Med. 2017;35:518-9.

14. Kriege M, Alflen C, Tzanova I, Schmidtmann I, Piepho T, Noppens RR. Evaluation of the McGrath MAC and Macintosh laryngoscope for tracheal intubation in 2000 patients undergoing general anaesthesia: the randomised multicentre EMMA trial study protocol. BMJ Open. 2017;7:e016907.

15. Janz DR, Semler MW, Lentz RJ, Matthews DT, Assad TR, Norman BC, et al. Randomized trial of video laryngoscopy for endotracheal intubation of critically ill adults. Crit Care Med. 2016;44:1980-7.

16. Smereka J, Madziala M, Dunder D, Makomaska-Szaroszyk E, Szarpak L. Comparison of Miller laryngoscope and UEScope videolaryngoscope for endotracheal intubation in four pediatric airway scenarios: a randomized, crossover simulation trial. Eur J Pediatr. 2019;178:937-45.

17. Smereka J, Czyzewski L, Szarpak L, Ladny JR. Comparison between the TrueView EVO2 PCD and direct laryngoscopy for endotracheal intubation performed by paramedics: Preliminary data. Am J Emerg Med. 2017;35:78990.

18. Szarpak Ł, Czyzewski Ł, Truszewski Z, Kurowski A, Gaszyzski T. Comparison of Coopdech $^{\circledR}$, CoPilot ${ }^{\circledR}$, Intubrite ${ }^{\circledR}$, and Macintosh laryngoscopes for tracheal intubation during pediatric cardiopulmonary resuscitation: a randomized, controlled crossover simulation trial. Eur J Pediatr. 2015;174:1517-23.

19. Szarpak $Ł$, Czyzewski $Ł$, Kurowski A. Comparison of the Pentax, Truview, Glidescope, and the Miller laryngoscope for child intubation during resuscitation. Am J Emerg Med. 2015;33:391-5.

20. Glosser L. Assessment of endotracheal tube intubation. Review of existing scales. Disaster Emerg Med J. 2017;2:91-3.

21. Szarpak L, Madziala A, Czekajlo M, Smereka J, Kaserer A, Dabrowski M, et al. Comparison of the UEScope videolaryngoscope with the Macintosh laryngoscope during simulated cardiopulmonary resuscitation: A randomized, cross-over, multi-center manikin study. Medicine (Baltimore). 2018;97:e12085.

22. Szarpak L, Czyzewski L, Kurowski A. Can GlideScope ${ }^{\circledR}$ videolaryngoscope be an alternative to direct laryngoscopy for child and infant tracheal intubation during chest compression? Eur J Pediatr. 2015;174:981-2. 
23. Savino PB, Reichelderfer S, Mercer MP, Wang RC, Sporer KA. Direct Versus Video Laryngoscopy for Prehospital Intubation: A Systematic Review and Meta-analysis. Acad Emerg Med. 2017;24:1018-26.

24. Hodnick R, Zitek T, Galster K, Johnson S, Bledsoe B, Ebbs D. A Comparison of Paramedic First Pass Endotracheal Intubation Success Rate of the VividTrac VT-A 100, GlideScope Ranger, and Direct Laryngoscopy Under Simulated Prehospital Cervical Spinal Immobilization Conditions in a Cadaveric Model. Prehosp Disaster Med. 2017;32:621-4.

25. Gawlowski P, Smereka J, Madziala M, Cohen B, Ruetzler K, Szarpak L. Comparison of the ETView Single Lumen and Macintosh laryngoscopes for endotracheal intubation in an airway manikin with immobilized cervical spine by novice paramedics: A randomized crossover manikin trial. Medicine (Baltimore). 2017;96:e5873.

26. Liu ZJ, Yi J, Guo WJ, Ma C, Huang YG. Comparison of McGrath series 3 and Macintosh laryngoscopes for tracheal intubation in patients with normal airway by inexperienced anesthetists: a randomized study. Medicine. 2016;95:e2514.

27. Walker L, Brampton W, Halai M, Hoy C, Lee E, Scott I, et al. Randomized controlled trial of intubation with the McGrath series 5 videolaryngoscope by inexperienced anaesthetists. Br J Anaesth. 2009;103:440-5.

28. Kaki AM, Almarakbi WA, Fawzi HM, Boker AM. Use of Airtraq, C-Mac, and Glidescope laryngoscope is better than Macintosh in novice medical students' hands: a manikin study. Saudi J Anaesth. 2011;5:376-81.

29. McElwain J, Malik MA, Harte BH, Flynn NM, Laffey JG. Comparison of the C-MAC videolaryngoscope with the Macintosh, Glidescope, and Airtraq laryngoscopes in easy and difficult laryngoscopy scenarios in manikins. Anaesthesia. 2010;65:483-9.

30. Szarpak L, Smereka J, Truszewski Z, Ruetzler K. Can novice physicians intubate with ETView tube without Macintosh laryngoscope? Preliminary data. Am J Emerg Med. 2016;34:2242-3.

31. Hoshijima H, Mihara T, Maruyama K, Denawa Y, Mizuta K, Shiga T, et al. C-MAC videolaryngoscope versus Macintosh laryngoscope for tracheal intubation: A systematic review and meta-analysis with trial sequential analysis. J Clin Anesth. 2018;49:53-62.

32. Owada G, Mihara T, Inagawa G, Asakura A, Goto T, Ka K. A comparison of the Airtraq ${ }^{\circledR}$, McGrath $^{\circledR}$, and Macintosh laryngoscopes for difficult paediatric intubation: a manikin study. PLoS One. 2017;12:e0171889.
33. Yoo JY, Park SY, Kim JY, Kim M, Haam SJ, Kim DH. Comparison of the McGrath videolaryngoscope and the Macintosh laryngoscope for double lumen endobronchial tube intubation in patients with manual in-line stabilization: a randomized controlled trial. Medicine. 2018;97:e0081.

34. Bernhard M, Mohr S, Weigand MA, Martin E, Walther A. Developing the skill of endotracheal intubation: implication for emergency medicine. Acta Anaesthesiol Scand. 2012;56:164-71.

35. De Oliveira Filho GR. The construction of learning curves for basic skills in anesthetic procedures: an application for the cumulative sum method. Anesth Analg. 2002;95:411-6.

36. Je S, Cho Y, Choi HJ, Kang B, Lim T, Kang H. An application of the learning curve-cumulative summation test to evaluate training for endotracheal intubation in emergency medicine. Emerg Med J. 2015;32:291-4.

37. Baciarello M, Zasa M, Manferdini ME, Tosi M, Berti M, Fanelli G. The learning curve for laryngoscopy: Airtraq versus Macintosh laryngoscopes. J Anesth. 2012;26:516-24.

38. Aghamohammadi H, Massoudi N, Fathi M, Jaffari A, Gharaei B, Moshki A. Intubation learning curve: comparison between video and direct laryngoscopy by inexperienced students. J Med Life. 2015;8:150-3.

39. Madziala M, Smereka J, Dabrowski M, Leung S, Ruetzler K, Szarpak L. A comparison of McGrath MAC ${ }^{\circledR}$ and standard direct laryngoscopy in simulated immobilized cervical spine pediatric intubation: a manikin study. Eur J Pediatr. 2017;176:779-86.

40. Frass M, Robak O, Truszewski Z, Czyzewski L, Szarpak L. Comparison of endotracheal intubation with the AirTraq Avant ${ }^{\circledR}$ and the Macintosh laryngoscope during intermittent or continuous chest compression: a randomized, crossover study in manikins. Disaster Emerg Med J. 2016;1:7-13.

41. Abelson A. Learning through simulation. Disaster Emerg Med J. 2017;2:125-8.

42. Czekajlo M, Dabrowska A. In situ simulation of cardiac arrest. Disaster Emerg Med J. 2017;2:116-9.

43. Smereka J, Ladny JR, Naylor A, Ruetzler K, Szarpak L. C-MAC compared with direct laryngoscopy for intubation in patients with cervical spine immobilization: a manikin trial. Am J Emerg Med. 2017;35:1142-6. 